\title{
Efecto de sistemas de sangría sobre la productividad de Hevea brasiliensis en el Magdalena Medio Santandereano
}

\section{Rubber tapping system effect on the productivity of Hevea brasiliensis in the Colombian Middle Magdalena}

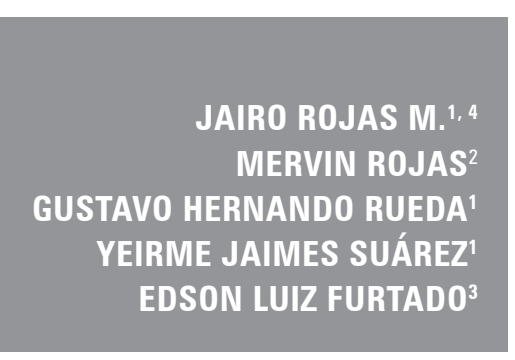

Sistema de sangría en caucho.

Foto: J. Rojas M.

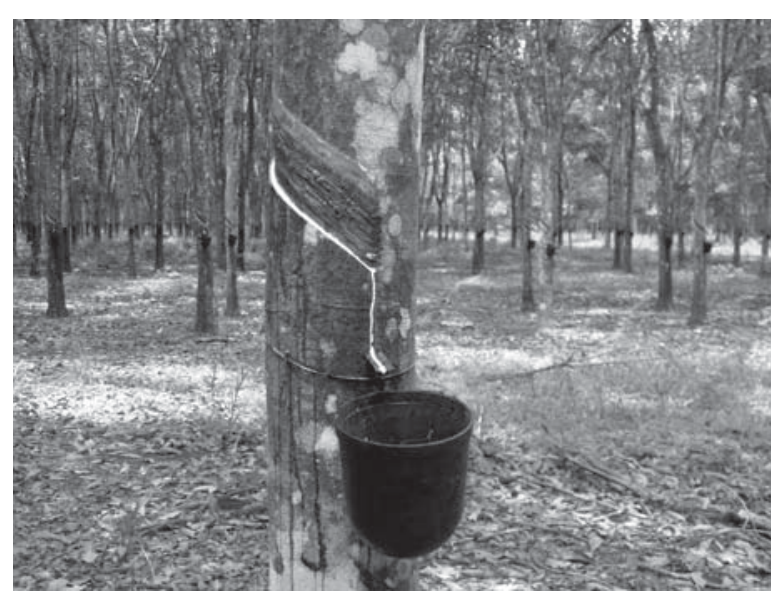

\section{RESUMEN}

Los sistemas de sangría con estimulación se usan para minimizar costos y aumentar la producción de látex. Por ello, se evaluaron sistemas de sangría con diferentes frecuencias con y sin estimulación con Ethrel® y niveles de fertilización en un cultivo del FX3864 de Hevea brasiliensis de 9 años de edad en Barrancabermeja, Santander. El estudio se realizó con un diseño de parcelas divididas distribuido en bloques al azar, considerando como parcela principal los sistemas de sangría con diferentes frecuencias con y sin estimulación y como subparcela los tratamientos de fertilización con dosis combinadas de N, P y K. La producción de caucho seco, consumo de corteza, incidencia de seca fisiológica, contenido de caucho seco y la rentabilidad económica se consideraron como variables de respuesta. La producción más alta se obtuvó con los sistemas d/3 E y d/4 E (1.446 y $1.281 \mathrm{~kg} \mathrm{ha}^{-1}$ año $^{-1}$, respectivamente), con diferencias significativas con los sistemas d/3 S.E y d/5 E. Entre los tratamientos de fertilización no se encontró diferencias significativas, aunque las mayores producciones se obtuvieron con los niveles 40-40-40 kg ha-1 y 80-80-80 kg ha-1 de N, P y K. En cuanto a los mayores niveles foliares de $\mathrm{N}, \mathrm{P}, \mathrm{K}$ y Ca se encontraron en el sistema d/4 E. Con estos resultados se recomienda el sistema de sangría d/4 E por tener la mejor rentabilidad económica bajo diferentes rendimientos del sangrador, menor consumo de corteza $(3,86 \mathrm{~cm})$, mayor vida útil de la plantación $(3,85$ años) y menor incidencia de enfermedades de panel.

\footnotetext{
Palabras clave adicionales: Ethrel, látex, producción, secado panel, fertilización.

1 Corporación Colombiana de Investigación Agropecuaria (Corpoica), Centro de Investigación La Suiza, Rionegro, Santander (Colombia).

2 Instituto Universitario La Paz, Escuela Ingeniería Agronómica, Barrancabermeja (Colombia).

3 Faculdade de Ciências Agronômicas, Departamento de Proteção de Plantas, Universidade Estadual de São Paulo, Botucatu, SP (Brasil) y Bolsista do CNPq.

4 Autor para correspondência.jrojas@corpoica.org.co
} 


\section{ABSTRACT}

Tapping systems with stimulation are used to minimize costs and improve latex yield. For this, tapping systems with different frequencies and with or without Ethrel ${ }^{\circledR}$ stimulation and fertilizer levels were evaluated in a 9-yearold crop of FX3864 of Hevea brasiliensis in Barrancabermeja, Santander. In this study, a split plot design with randomized complete blocks was used, where the tapping systems with different frequencies with or without stimulation were considered as the principal plot and the fertilization treatments with combined dosages of $\mathrm{N}, \mathrm{P}$ and $\mathrm{K}$ were considered as the subplots. The dry rubber yield, bark consumption, tapping panel dryness incidence, dry rubber content and profitability were considered as response variables. The systems $\mathrm{d} / 3 \mathrm{E}$ and $\mathrm{d} / 4 \mathrm{E}$ obtained the highest yield (1,446 and 1,281 $\mathrm{kg} \mathrm{ha}^{-1} \mathrm{yr}^{-1}$, respectively), with significant differences with the systems d/3 S.E and d/5 E. For the fertilization treatments, there were no significant differences. Notwithstanding, the highest yields were obtained in the 40-40-40 $\mathrm{kg} \mathrm{ha}^{-1}$ and 80-80-80 $\mathrm{kg} \mathrm{ha}^{-1}$ of N, P and $\mathrm{K}$ balanced treatments. The higher foliar levels of N, P, K and $\mathrm{Ca}$ were founded in the $\mathrm{d} / 4 \mathrm{E}$ system. According to these results, the $\mathrm{d} / 4 \mathrm{E}$ tapping system is recommended to be the most profitable under different tapping yields, with less bark consumption $(3.86 \mathrm{~cm})$, longer life plantation (3.85 years) and less disease panel incidence.

Addicional key words: Ethrel stimulation, látex, yield, panel dryness, fertilization.

Fecha de recepción: 24-06-2016 Aprobado para publicación: 15-04-2017

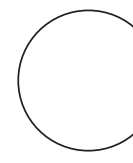

En la actualidad, el árbol de caucho, Hevea brasiliensis (Willd. ex Adr. de Juss.) Muell. Arg., se considera una de las principales fuentes de caucho natural en el mundo. El caucho natural es una materia prima estratégica para más de 40 mil productos de gran importancia principalmente en las industrias automotriz (neumáticos) y dispositivos médicos entre otros usos. Debido a la tendencia mundial hacia el incremento de los costos de producción y la poca disponibilidad de la mano de obra, se continúa investigando sobre los métodos que permitan reducir los costos de producción en las plantaciones de caucho (Silva et al., 2010). La cosecha del látex se considera la operación de mayor costo en las plantaciones comerciales. Para su ejecución se requiere de un gran nivel de habilidades y esto junto con los bajos salarios lleva a la escasez de sangradores calificados. Este problema se agrava con las condiciones ambientales desfavorables, días de trabajo interrumpidos, pobres condiciones del suelo, instalaciones de infraestructura inadecuadas y carencia de programas de entrenamiento (Rodrigo et al., 2011).

Con todo, una de las mayores dificultades radica en la definición de los sistemas de sangría que pueden generar mejores producciones sin comprometer el crecimiento vegetativo, la fisiología y la salud de la planta. La explotación o sangría es fundamental en la determinación de la vida útil y producción del árbol de caucho. El mejoramiento de la producción se puede logra a través de la intensificación de la frecuencia de cosecha o mediante la estimulación química. En trabajos recientes con clones de metabolismo lento, especialmente el PB 217 y PR 107, se ha demostrado que la reducción de la frecuencia de sangría es la mejor alternativa a los sistemas de sangría tradicionales (Soumahin et al., 2010; Obouayeba et al., 2011; Diarrassouba et al., 2012). Sin embargo, con las bajas frecuencia se puede no alcanzar volúmenes apropiados de látex. Para atender a esta limitación, en los países productores de caucho se investiga sobre el uso de estimulantes, tal como el Ethephon o Ethrel, en combinación con bajas frecuencias de sangría que favorezcan la producción (Silva et al., 2010).

El Ethrel@ estimula el árbol de caucho para incrementar la producción de caucho y realza los procesos de regeneración del látex. Cuando éste se aplica sobre el área de corte del panel de sangría, se libera gas etileno incrementando la duración del flujo de látex mediante el retraso del taponamiento de las vesículas laticíferas. Para producciones óptimas, la frecuencia de la estimulación y la concentración del estimulante se debe modular en función del clon, edad del árbol y sistema de sangría (Njukeng et al., 2011). 
Adicionalmente, para mantener y estabilizar la producción en unos altos niveles durante la explotación o sangría, la fertilización mineral puede incrementar y mantener la productividad de caucho de manera sostenible. En efecto, las altas producciones resultan en una exportación significativa de minerales durante la sangría, por esto se requiere una corrección compensatoria mediante la fertilización para mantener el balance. Con esto se explica el hecho que en Malasia las entradas de fertilizantes sean sistemáticas y graduales a rendimientos superiores a $1.400 \mathrm{~kg} \mathrm{ha}^{-1}$ (Alle et al., 2015).

De acuerdo con lo expuesto, el objetivo del trabajo fue evaluar tres sistemas de sangría con estimulación y uno sin estimulación en un cultivo de caucho establecido en Barrancabermeja (Santander), así como niveles de fertilización que permitieran realzar y mantener la producción de látex.

\section{MATERIALES Y MÉTODOS}

\section{Sitio de estudio y material vegetal}

El ensayo se localizó en la finca El Divino Niño, ubicada en la vereda El Rodeo, municipio de Barrancabermeja (Santander), con coordenadas $07^{\circ} 10^{\prime} 47,7^{\prime \prime} \mathrm{N}$ y $073^{\circ} 50^{\prime} 00,2^{\prime \prime} \mathrm{W}$, precipitación media anual de 2.317 $\mathrm{mm}$, temperatura media anual de $28,7^{\circ} \mathrm{C}$ y humedad relativa media del $84 \%$ (Fig. 1). El estudio se hizo en árboles del clon FX 3864 con 9 años de edad en el tercer año de explotación comercial con $60 \pm 5 \mathrm{~cm}$ perímetro de circunferencia del tallo a 1,3 $\mathrm{m}$ a la altura del suelo o altura del pecho.

\section{Diseño experimental}

Se utilizó un diseño de parcelas sub-divididas en bloques completos al azar. Como parcela principal se consideró el sistema de sangría compuesto cada uno por 10 árboles y tres repeticiones. Las subparcelas se consideraron 10 tratamientos con niveles de fertilización con niveles de N, P y K variables y fijos los elementos menores.

\section{Sistemas de sangría (parcela principal)}

Para todos los sistemas de sangría se adoptó un corte en media espiral $(1 / 2 S)$ descendente, el cual se efectuó seis días de la semana ( 6 d/7). En los sistema de sangría en evaluación se modificó las frecuencias variando cada tres (d3), cuatro (d4) y cinco días (d/5) con aplicaciones de Ethrel ${ }^{\circledR}$ a una concentración de $2,5 \% \mathrm{v} \mathrm{v}^{-1}$ (ET 2,5\%). También se incluyó un tratamiento con frecuencia cada tres días $(\mathrm{d} / 3)$ sin estimulación. En los tratamientos con estimulación, el Ethrel® se aplicó sobre el área de sangría en 2,5\% de ingrediente activo. Éste se aplicó por pincelado y aplicado sobre el canal de corte (La) cuatro veces en el año (4/y) (Tab. 1).

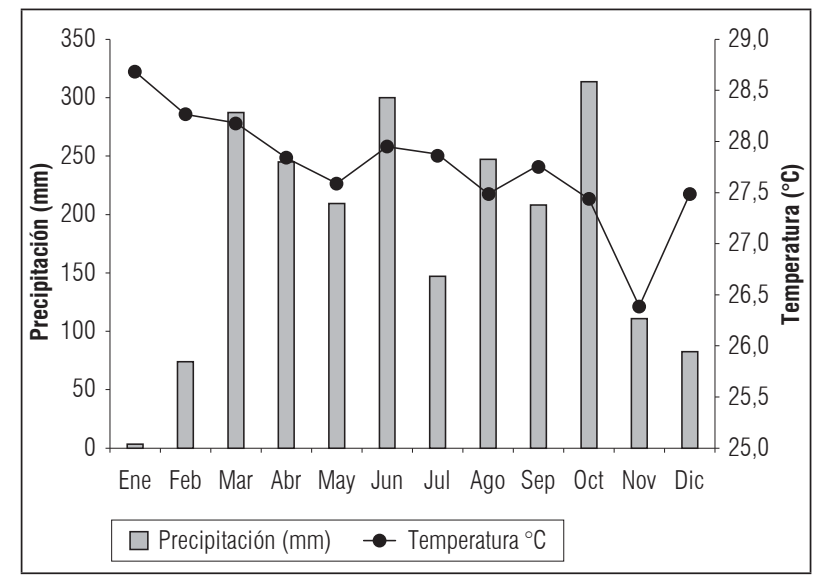

Figura 1. Precipitación y temperatura media mensual en la finca El Divino Niño ubicada en Barrancabermeja (Santander) entre enero y diciembre de 2014.

\section{Plan de fertilización (subparcela)}

Diferentes niveles de NPK se evaluaron para cada sistema de sangría (Tab. 1). Dónde: $0=$ no aplicación del elemento; $1=40 \mathrm{~kg} \mathrm{ha}^{-1}$ del elemento; $2=80 \mathrm{~kg} \mathrm{ha}^{-1}$ del elemento. Se utilizaron como fuentes: urea para $\mathrm{N}(46 \% \mathrm{~N})$, DAP (fosfato diamónico) (18\% N y 46\% $\left.\mathrm{P}_{2} \mathrm{O}_{5}\right)$ para $\mathrm{P}$ y $\mathrm{KCl}\left(60 \% \mathrm{~K}_{2} \mathrm{O}\right)$ como fuente potásica Todos los árboles del ensayó se fertilizaron con $30 \mathrm{~g}$ $\mathrm{MgSO}_{4}, 5 \mathrm{~g}$ de Boro agrícola y 2,5 g de $\mathrm{ZnSO}_{4}$.

Durante 10 meses, se realizaron muestreos de fondo de taza de cada uno de los sistemas de sangría utilizados dentro de la investigación. Los coágulos obtenidos después de la aplicación de ácido fórmico (5\%) se secaron durante 15 a 20 d. Esta actividad se realizó en la infraestructura dispuesta por el productor para esta labor. Una vez el coagulo alcanzó peso constante se registró el peso y se calculó la productividad en gramos por árbol y se extrapoló la producción a ha año ${ }^{-1}$, utilizando 400 árboles activos. Además, se midió el 
Tabla 1. Sistemas de sangría y niveles de fertilización evaluados en plantación de caucho natural en la finca El Divino Niño ubicada en Barrancabermeja (Santander).

\begin{tabular}{|c|c|c|c|c|}
\hline \multicolumn{3}{|c|}{ Parcela principal } & \multirow{3}{*}{\multicolumn{2}{|c|}{$\begin{array}{c}\text { Sub-parcela } \\
\text { Dosis de fertilizantes }^{1}\end{array}$}} \\
\hline \multicolumn{3}{|c|}{ Sistema de sangría } & & \\
\hline Tratamiento & Código & el sistema & & \\
\hline \multirow{5}{*}{$\mathrm{d} / 3 \mathrm{~S} . \mathrm{E}$} & \multirow{5}{*}{$1 / 2 \mathrm{~S} \mathrm{~d} / 36 \mathrm{~d} / 7$} & \multirow{5}{*}{$\begin{array}{l}\text { Corte de medio espiral descendente con frecuencia } \\
\text { de } 3 d, 6 \text { d de sangría seguida por } 1 d \text { de descanso, } \\
\text { sin estimulación. } \\
\text { T3: N1P1K1 } \\
\text { T5: N1P2K0 } \\
\text { T7: N1P2K2 } \\
\text { T9: N2P1K1 }\end{array}$} & T1: NOPOKO & T2: N1P1K0 \\
\hline & & & T4: N1P1K2 & \\
\hline & & & T6: N1P2K1 & \\
\hline & & & T8: N2POKO & \\
\hline & & & T10:N2P2K2 & \\
\hline \multirow{5}{*}{$d / 3 E$} & \multirow{5}{*}{ 1/2S d/3 6d/7 ET 2,5\% 4/y } & \multirow{5}{*}{$\begin{array}{l}\text { Corte de medio espiral descendente con frecuencia } \\
\text { de } 3 d, 6 \text { d de sangría seguida por } 1 \mathrm{~d} \text { de descanso, } \\
\text { estimulada con Ethephon de } 2,5 \% \text { de ingrediente } \\
\text { activo y cuatro aplicaciones en el año. } \\
\text { T3: N1P1K1 } \\
\text { T5: N1P2K0 } \\
\text { T7: N1P2K2 } \\
\text { T9: N2P1K1 }\end{array}$} & T1: NOPOKO & T2: N1P1K0 \\
\hline & & & T4: N1P1K2 & \\
\hline & & & T6: N1P2K1 & \\
\hline & & & T8: N2POKO & \\
\hline & & & T10:N2P2K2 & \\
\hline \multirow{5}{*}{$d / 4 E$} & \multirow{5}{*}{ 1/2S d/4 6d/7 ET 2,5\% 4/y } & \multirow{5}{*}{$\begin{array}{l}\text { Corte de medio espiral descendente con frecuencia } \\
\text { de } 4 \mathrm{~d}, 6 \mathrm{~d} \text { de sangría seguida por } 1 \mathrm{~d} \text { de descanso, } \\
\text { estimulada con Ethephon de } 2,5 \% \text { de ingrediente } \\
\text { activo y cuatro aplicaciones en el año. } \\
\text { T3: N1P1K1 } \\
\text { T5: N1P2K0 } \\
\text { T7: N1P2K2 } \\
\text { T9: N2P1K1 }\end{array}$} & T1: NOPOKO & T2: N1P1K0 \\
\hline & & & T4: N1P1K2 & \\
\hline & & & T6: N1P2K1 & \\
\hline & & & T8: N2POKO & \\
\hline & & & T10:N2P2K2 & \\
\hline \multirow{5}{*}{$d / 5 E$} & \multirow{5}{*}{$1 / 2 \mathrm{~S} \mathrm{~d} / 5 \mathrm{6d} / 7 \mathrm{ET} 2,5 \% 4 / \mathrm{y}$} & \multirow{5}{*}{$\begin{array}{l}\text { Corte de medio espiral descendente con frecuencia } \\
\text { de } 5 d, 6 \text { d de sangría seguida por } 1 \mathrm{~d} \text { de descanso, } \\
\text { estimulada con Ethephon de } 2,5 \% \text { de ingrediente } \\
\text { activo y cuatro aplicaciones en el año. } \\
\text { T3: N1P1K1 } \\
\text { T5: N1P2K0 } \\
\text { T7: N1P2K2 } \\
\text { T9: N2P1K1 }\end{array}$} & T1: NOPOKO & T2: N1P1K0 \\
\hline & & & T4: N1P1K2 & \\
\hline & & & T6: N1P2K1 & \\
\hline & & & T8: N2POKO & \\
\hline & & & T10:N2P2K2 & \\
\hline
\end{tabular}

10: no aplicación del elemento; 1: $40 \mathrm{~kg} \mathrm{ha-1}$ del elemento; 2: $80 \mathrm{~kg} \mathrm{ha}^{-1}$ del elemento.

consumo de corteza cada mes, así como se registró la incidencia de seca de panel y enfermedades de panel para cada sistema de sangría en evaluación.

Dentro de los parámetros bioquímicos, se midió el contenido de caucho seco (DRC) cada mes. Para esto se tomó $200 \mathrm{~cm}^{3}$ de látex por cada sistema y mediante un densímetro convencional 700:1.000 se obtuvo la densidad de látex por tratamiento por repetición. Posteriormente, las muestras se pesaron, laminaron y secaron hasta peso constante. Este proceso requirió entre 12 a 15 d. El análisis económico se calculó con los datos de producción y de costo de mano de obra de cada uno de los sistemas de sangría implementados bajo diferentes rendimientos de número de árboles sangrados/día.

\section{Análisis estadístico}

Los análisis estadisticos se hicieron con el programa Infostat, versión 2013 (Di Rienzo et al., 2015). Para esto, se realizó un ANOVA para la variables de producción y consumo de corteza bajo los diferentes sistemas de sangría, cuando se encontró diferencia entre tratamiento se realizó una prueba Duncan al 95\% de confianza. 


\section{RESULTADOS Y DISCUSIÓN}

Con el sistema 1/2S d/3 6d/7 ET 2,5\% 4/y se obtuvo los mejores rendimientos con diferencia estadística significativa con el sistema $1 / 2 \mathrm{~S} \mathrm{~d} / 36 \mathrm{~d} / 7$ sin estimulación y el sistema $1 / 2 \mathrm{~S} \mathrm{~d} / 56 \mathrm{~d} / 7 \mathrm{ET} 2,5 \% 4 / \mathrm{y}$, pero no difiere significativamente del sistema $1 / 2 \mathrm{~S} \mathrm{~d} / 46 \mathrm{~d} / 7$ ET 2,5\% 4/y. Entre los sistemas 1/2S d/5 6d/7 ET $2,5 \% 4 / y$ y el sistema $1 / 2 \mathrm{~S} d / 36 \mathrm{~d} / 7$ sin estimulación no se obtuvo diferencias significativas (Tab. 2). Estos resultados coinciden con los estudios de Njukeng et al. (2011) y Silva et al. (2010). En el primer estudio se encontró que la aplicación de Ethrel®, cuando comparado con tratamientos sin aplicación de estimulantes, permite incrementar significativamente las producciones anuales para los clones evaluados. Cuando los autores compararon con la aplicación de Ethrel ${ }^{\circledR}$ cuatro veces al año con la no aplicación de estimulante, se obtuvieron producciones muy superiores en los clones GT 1, IRCA 19 y PB 260.

En el estudio de Silva et al. (2010) se reportaron que los sistemas de sangría 1/2S d/3 6d/7 ET 2,5\% y $1 / 2 \mathrm{~S} d / 46 \mathrm{~d} / 7$ ET $2,5 \%$ proveen alta producción y rentabilidad por hectárea por año y baja incidencia de seca de panel de sangría comparado con tratamientos con Ethrel ${ }^{\circledR}$ 5\% para los clones RRIM 600 y PR 261. En cuanto a la producción superior para los clones IAN 873 y RRIM 703 se presentó con el sistema $1 / 2 \mathrm{~S} \mathrm{~d} / 36 \mathrm{~d} / 7$ ET $2,5 \%$ de alta frecuencia de sangría. Sin embargo, en este estudio la mejor rentabilidad se obtuvo con el sistema 1/2S d/7 6d/7 ET $2,5 \%$ de baja frecuencia de sangría (Silva et al, 2010) El vigor de la planta en el clon IAN 873 se vió más afectado por la sangría con subsecuente disminución del incremento de circunferencia. La estimulación de látex con Ethephon 5\% mostró una tendencia por decrecer la producción por hectárea por año y favoreció alta incidencia del panel de sangría seco.
Cabe decir que intervalos de $4 \mathrm{~d}$ entre sangrías se han reportado como los requeridos y adecuados para la reconstitución del contenido del látex en las vesículas de látex. En intervalos menores a $4 \mathrm{~d}$ se presenta un rápido incremento de los sólidos totales, mientras que la producción decrece rápidamente, cuando comparados a intervalos superiores a los $7 \mathrm{~d}$. Las frecuencias de 4 y $5 \mathrm{~d}$ pueden permitir mejor uso de los factores de producción, pero la reducción de cortes puede decrecer la productividad a niveles no económicos. Consecuentemente, los clones que muestran mejor respuesta a la estimulación y proveen una producción alta y sustentable se prefieren como alternativa para disminuir las frecuencias de sangría (Silva et al., 2010).

En cuanto a las mejores producciones de caucho seco por hectárea por sangría se obtuvieron con los sistemas de frecuencias bajas (sistema 1/2S d/3 6d/7 ET $2,5 \%$ 4/y y sistema 1/2S d/4 6d/7 ET 2,5\% 4/y) (Tab. 2). Soumahin et al. (2009) observaron que la producción es más alta cuando los árboles de caucho se estimulan usando Ethrel ${ }^{\circledR}$ al $2,5 \%$ y no al $5 \%$, por ende siendo más efectivo al 2,5\%. Un incremento en la concentración de ingrediente activo puede tornarse tóxico para los árboles, posiblemente debido al efecto de sobredosis. Así, con la concentración al 2,5\% se considera la mejor opción para compensar la baja frecuencia de sangría, y se prefiere aumentar la frecuencia de estimulación antes de aumentar la concentración del ingrediente activo.

Los valores altos de consumo de corteza de sangría se obtuvieron con las frecuencias altas de los sistemas $1 / 2 \mathrm{~S} d / 36 \mathrm{~d} / 7$ sin estimulación y $1 / 2 \mathrm{~S} d / 36 \mathrm{~d} / 7 \mathrm{ET}$ $2,5 \% 4 / y$ con diferencia significativa con el sistema $1 / 2 \mathrm{~S} \mathrm{~d} / 46 \mathrm{~d} / 7$ ET $2,5 \%$ 4/y y altamente significativo del sistema $1 / 2 \mathrm{~S} \mathrm{~d} / 5$ 6d/7 ET 2,5\% 4/y (Tab. 3). Con base en estos resultados se calculó la vida útil de la cara A del árbol para cada sistema, destacándose los sistemas con frecuencia d/5 y d/6 con 3,85 y 4,96

Tabla 2. Producción de caucho seco por árbol en diferentes sistemas de sangría en la finca El Divino Niño ubicada en el municipio de Barrancabermeja (Santander).

\begin{tabular}{|c|c|c|}
\hline Sistema de sangría & $\begin{array}{c}\text { Producción media } \\
\pm \text { error estándar (g/árbol-año) }\end{array}$ & $\begin{array}{c}\text { Producción } \\
\left(\mathrm{kg} \mathrm{h}^{-1} \text { año-1 }^{-1}\right)\end{array}$ \\
\hline $1 / 2 \mathrm{~S} \mathrm{~d} / 3 \mathrm{dd} / 7$ & $2.463,67 \pm 136 \mathrm{bc}$ & 1108 \\
\hline $1 / 2 \mathrm{~S} \mathrm{~d} / 3 \mathrm{~d} / 7 \mathrm{ET} 2,5 \% 4 / \mathrm{y}$ & $3.214,93 \pm 136 \mathrm{a}$ & 1281 \\
\hline $1 / 2 \mathrm{~S} \mathrm{~d} / 46 \mathrm{~d} / 7 \mathrm{ET} 2,5 \% 4 / \mathrm{y}$ & $2.847,47 \pm 136 \mathrm{ab}$ & 1042 \\
\hline $1 / 2 \mathrm{~S} \mathrm{~d} / 5 \mathrm{~d} / 7 \mathrm{ET} 2,5 \% 4 / \mathrm{y}$ & $2.317,7 \pm 136 \mathrm{c}$ \\
\hline
\end{tabular}

Promedios con letras distintas indican diferencia significativa según la prueba de Duncan $(P \leq 0,05)$. 
Tabla 3. Vida útil de la cara A del panel de sangría de acuerdo al consumo de corteza en los diferentes sistemas de sangría en la finca El Divino Niño ubicada en Barrancabermeja (Santander).

\begin{tabular}{|c|c|c|}
\hline Sistema de sangría & $\begin{array}{c}\text { Media } \pm \text { error } \\
\text { estándar } \\
\text { (cm) }\end{array}$ & $\begin{array}{c}\text { Vida útil cara } \\
\text { A (años) }\end{array}$ \\
\hline $1 / 2 \mathrm{~S} \mathrm{~d} / 3 \mathrm{dd} / 7$ & $29,95 \pm 0,22 \mathrm{c}$ & $3,04(100)$ \\
\hline $1 / 2 \mathrm{~S} \mathrm{~d} / 36 \mathrm{~d} / 7 \mathrm{ET} 2,5 \% 4 / \mathrm{y}$ & $29,50 \pm 0,22 \mathrm{c}$ & $3,08(101,3)$ \\
\hline $1 / 2 \mathrm{~S} d / 46 \mathrm{~d} / 7 \mathrm{ET} 2,5 \% 4 / \mathrm{y}$ & $23,63 \pm 0,22 \mathrm{~b}$ & $3,85(126,64)$ \\
\hline $1 / 2 \mathrm{~S} \mathrm{~d} / 5 \mathrm{6d} / 7 \mathrm{ET} 2,5 \% 4 / \mathrm{y}$ & $18,36 \pm 0,22 \mathrm{a}$ & $4,96(163,15)$ \\
\hline
\end{tabular}

Promedios con letras distintas indican diferencia significativa según la prueba de Duncan $(P \leq 0,05)$.

Tabla 4. Consumos de corteza en sistemas de sangría en Barrancabermeja (Santander).

\begin{tabular}{|c|c|}
\hline Sistema de sangría & Media (cm) \\
\hline $1 / 2 \mathrm{sd} / 3^{*} 6 \mathrm{~d} / 7$ & $29,95 \mathrm{c}$ \\
\hline $1 / 2 \mathrm{sd} / 36 \mathrm{~d} / 7 \mathrm{7} / \mathrm{y} \mathrm{ET} 2,5 \%$ & $29,5 \mathrm{c}$ \\
\hline $1 / 2 \mathrm{sd} / 46 \mathrm{~d} / 7$ 4/y ET $2,5 \%$ & $23,63 \mathrm{~b}$ \\
\hline $1 / 2 \mathrm{sd} / 56 \mathrm{~d} / 7$ 4/y ET 2,5\% & $18,36 \mathrm{a}$ \\
\hline
\end{tabular}

Promedios con letras distintas indican diferencia significativa según la prueba de Duncan $(P \leq 0,05)$.

Tabla 5. Vida útil de Cara A del panel de sangría con base en consumos de corteza de los sistemas de sangría en Barrancabermeja (Santander).

\begin{tabular}{|c|c|c|}
\hline Sistema de sangría & $\begin{array}{c}\text { Consumo de } \\
\text { corteza }\end{array}$ & $\begin{array}{c}\text { Vida útil Cara A } \\
\text { (Años) }\end{array}$ \\
\hline $1 / 2 \mathrm{~s} \mathrm{~d} / 3^{*} 6 \mathrm{~d} / 7$ & $29,95 \mathrm{~cm}$ & 3,04 \\
\hline $1 / 2 \mathrm{~s} \mathrm{~d} / 36 \mathrm{~d} / 74 / \mathrm{y} \mathrm{ET} \mathrm{2,5 \%}$ & $29,5 \mathrm{~cm}$ & 3,08 \\
\hline $1 / 2 \mathrm{~s} \mathrm{~d} / 46 \mathrm{~d} / 74 / \mathrm{y} \mathrm{ET} \mathrm{2,5 \%}$ & $23,63 \mathrm{~cm}$ & 3,85 \\
\hline $1 / 2 \mathrm{~s} \mathrm{~d} / 56 \mathrm{~d} / 74 / \mathrm{y} \mathrm{ET} 2,5 \%$ & $18,46 \mathrm{~cm}$ & 4,96 \\
\hline
\end{tabular}

años, respectivamente (Tab. 3). Los consumos altos se presentaron por falta de capacitación del sangrador, lo que se mejoró al final del experimento con instrucción continua por el grupo de trabajo.

Silva et al. (2010) encontraron que la reducción en el consumo de corteza es benéfica, porque aumenta la vida útil de los árboles y permite una mayor regeneración del sistema laticífero. Por ello, en los trabajos desarrollados por Silva et al. (2010) se observó que la reducción en el consumo de corteza se hace con frecuencias bajas de sangría y esto hace que se prolongue la vida útil de las plantaciones (Tab. 4). En el presente estudio se encontró que al utilizar frecuencias bajas el consumo se reduce hasta en un $38 \%$, provocando que se utilice el panel de sangría en dos años más (Tab. 5). Esto último se obtuvo proyectando la vida útil del panel de sangría con los valores de consumo de corteza. Así, con esta información se evidenció que al emplear frecuencias de sangría baja se aumenta el periodo de explotación de la plantación al reducir el número de veces que sangra el rayador (Tab. 5).

Se presentó baja incidencia de seca fisiológica sobre los sistemas estimulas con Ethrel 2,5\% (Tab. 6), la mayor incidencia se presentó sobre el sistema de sangría que no se estimularon y por ende puede estar relacionado con el material de siembra utilizado y no con el uso de los estimulantes. Según Gonçalves et al. (2000), este disturbio puede presentarse cuando se utilizan sistemas de explotación de alta frecuencia y altas concentraciones de estimulante.

En cuanto al contenido de DRC, se obtuvieron valores altos para todos los sistemas de sangría, siendo buen indicador de la calidad de látex producido (Tab. 7). Cabe decir que con los sistemas de sangría d/3 E y d/3 S.E se obtuvieron los mejores porcentajes de DRC cuando comparado con los sistemas que involucran estimulación. Phakagrong et al. (2010) observaron que la estimulación decrece los contenidos de DRC en el látex entre 2 y $7 \%$ como un balance entre la biosíntesis del caucho y la extracción. Esta caída del DRC decrece la viscosidad del látex lo cual resulta en un

Tabla 6. Incidencia de seca de panel en ensayo de sangría en la Finca El Divino Niño ubicada en Barrancabermeja (Santander).

\begin{tabular}{|c|c|c|c|}
\hline Sistema de sangría & No. de plantas sangradas & No. de plantas con seca fisiológica & Seca fisiológica (\%) \\
\hline $1 / 2 S d / 36 d / y$ & 30 & 2 & 6,6 \\
\hline $1 / 2 S d / 36 d / 7$ ET 2,5\% 4/y & 30 & 0 & 0 \\
\hline $1 / 2 S d / 46 d / 7$ ET 2,5\% 4/y & 30 & 1 & 3,3 \\
\hline $1 / 2 S d / 56 d / 7$ ET 2,5\% 4/y & 30 & 0 & 0 \\
\hline
\end{tabular}


incremento de la tasa del flujo inicial y la duración del flujo. Sin embargo, a medida que se reduce la plasticidad inicial se incrementa el índice de retención de plasticidad. Así, la estimulación con Ethephon puede no solo incrementar la productividad sino que también tiende a mejorar las propiedades físicas del caucho.

Tabla 7. Contenido de DRC de látex obtenido bajo diferentes sistemas de sangría en la finca El Divino Niño ubicada en Barrancabermeja (Santander).

\begin{tabular}{|c|c|}
\hline Sistema de sangría & $\begin{array}{c}\text { Media } \pm \text { error estándar } \\
(\%)\end{array}$ \\
\hline $1 / 2 \mathrm{~S} \mathrm{~d} / 36 \mathrm{~d} / 7$ & $44,16 \pm 1,09 \mathrm{a}$ \\
\hline $1 / 2 \mathrm{~S} \mathrm{~d} / 36 \mathrm{~d} / 7 \mathrm{ET} 2,5 \% 4 / \mathrm{y}$ & $40,24 \pm 1,09 \mathrm{~b}$ \\
\hline $1 / 2 \mathrm{~S} \mathrm{~d} / 46 \mathrm{~d} / 7 \mathrm{ET} 2,5 \% 4 / \mathrm{y}$ & $41,39 \pm 1,09 \mathrm{ab}$ \\
\hline $1 / 2 \mathrm{~S} \mathrm{~d} / 5 \mathrm{dd} / 7 \mathrm{ET} 2,5 \% 4 / \mathrm{y}$ & $40,71 \pm 1,09 \mathrm{ab}$ \\
\hline
\end{tabular}

Promedios con letras distintas indican diferencia significativa según la prueba de Duncan $(P \leq 0,05)$.

Con respecto a el efecto de la fertilización sobre la producción de caucho, no se obtuvieron diferencias significativas, (Tab. 8) aunque se obtuvieron tendencias a mejores extracciones cuando los niveles de $\mathrm{N}$, $\mathrm{P}, \mathrm{K}$ se encontraban equilibradas en T3: N1P1K1 y T10: N2P2K2; esto es $40 \mathrm{~kg} \mathrm{ha}^{-1}$ y $80 \mathrm{~kg} \mathrm{ha}^{-1}$. En el estudio de Virgens Filho et al. (2001) encontraron que la producción de caucho seco se aumenta con la aplicación de K en la presencia de N y P, destacándose el tratamiento N2P2K1 con encalamiento, así se obtuvieron ganancias de $9 ; 19,2$ y $24,7 \%$ en los primeros tres periodos evaluados. Dado que el incremento en el grosor de la corteza, cuando se considera el mismo clon, tiene correlación directa con la expansión de la corteza blanda, sitio en el que se encuentran los vasos laticíferos más jóvenes, funcionales y productivos. Bajo condiciones de un adecuado estado nutricional, se puede obtener un mayor espesor y más rápido de la corteza. Esto favorece las condiciones del panel para futuras sangrías. El incremento se considera de gran importancia en la explotación de los cultivos de caucho, posibilitando una mejor distribución del área de drenaje, proporcionando mayores producciones por planta. También, se permite una gestión más adecuada del panel, mejorando la posición del corte en la sangría, bien como, evitar el descenso del sangrado hasta el tronco y así mejorar el rendimiento de la mano de obra (Virgens Filho et al., 2003).

La utilización de sustancias estimulantes que permitan el aumento de flujo de látex por sangría tiene

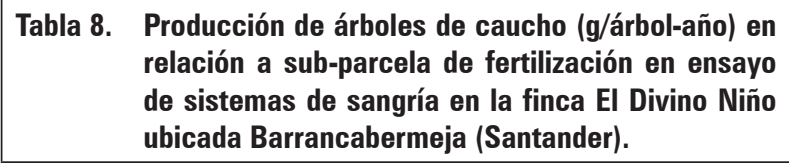

\begin{tabular}{|c|c|c|}
\hline Tratamientos & $\begin{array}{c}\text { Niveles de } \\
\text { fertilización }\end{array}$ & $\begin{array}{c}\text { Media } \pm \text { error estándar } \\
\text { (g) }\end{array}$ \\
\hline T1 & NOPOK0 & $2.760,58 \pm 216,45 \mathrm{a}$ \\
\hline T2 & N1P1K0 & $2.548,75 \pm 216,45 \mathrm{a}$ \\
\hline T3 & N1P1K1 & $2.93,08 \pm 216,45 \mathrm{a}$ \\
\hline T4 & N1P1K2 & $2.403,67 \pm 216,45 \mathrm{a}$ \\
\hline T5 & N1P2K0 & $2.702,58 \pm 216,45 \mathrm{a}$ \\
\hline T6 & N1P2K1 & $2.786,42 \pm 216,45 \mathrm{a}$ \\
\hline T7 & N1P2K2 & $2.760,92 \pm 216,45 \mathrm{a}$ \\
\hline T8 & N2POK0 & $2.623,75 \pm 216,45 \mathrm{a}$ \\
\hline T9 & N2P1K1 & $2.569,83 \pm 216,45 \mathrm{a}$ \\
\hline T10 & N2P2K2 & $2.959,83 \pm 216,45 \mathrm{a}$ \\
\hline
\end{tabular}

Promedios con letras distintas indican diferencia significativa según la prueba de Duncan $(P \leq 0,05)$.

compensación en el menor número de cortes en los sistemas de baja frecuencia, permitiendo lograr los dos factores básicos de la producción, fisiológico y económico. La reducción en la cantidad de sangrías disminuye el trauma de las plantas, aumentando el tiempo de regeneración del látex entre dos sangrías y reduce las enfermedades fisiológicas, tal como el secamiento del panel. Aún, permite una reducción importante y difícil que es la mano de obra especializada de los sangradores, con un aumento en la producción por unidad de área, mayor rendimiento financiero líquido, y menor consumo de corteza, aumentando así el período productivo de las plantas (Gonçalves et al., 2000).

Para la realización de la proyección económica, se utilizó como referencia los precios y los estándares de la Compañía Cauchera Colombiana de $\$ 2.900$ por kg $y$ con un DRC de $77 \%$. Se tuvieron en cuenta rendimientos por sangría de 450, 500, 600 y 700 árboles sangrados/día (Tab. 9).

La rentabilidad de los sistemas estimulados son mayores al sistemas tradicional d/3 S.E, el costo de la mano de obra ha ${ }^{-1}$ es menor en sistemas de menor frecuencia de sangría. La rentabilidad de los sistemas d/3 E y d/4 E son similares (Tab. 9), encontrándose que en cuanto al rendimiento del sangrador de 450 a 500 árboles por tarea el mejor sistema es d/3 E y a 
Tabla 9. Análisis económico de diferentes sistemas de sangría del clon Fx3864 establecido en la finca El Divino Niño ubicada en Barrancabermeja (Santander), con base en la producción de caucho seco por hectárea'.

\begin{tabular}{|c|c|c|c|c|c|c|c|}
\hline $\begin{array}{l}\text { Rendimiento } \\
\text { de sangrador }\end{array}$ & $\begin{array}{c}\text { Sistema } \\
\text { de sangría }\end{array}$ & Área (ha) & $\begin{array}{l}\text { Producción sangría } \\
\left(\mathrm{kg} \mathrm{ha}^{-1} \text { año-1 }\right)\end{array}$ & $\begin{array}{l}\text { Valor venta } \\
\text { bruto }\end{array}$ & $\begin{array}{c}\text { Costo } \\
\text { sangrador/ } \\
\text { ha-año }\end{array}$ & $\begin{array}{l}\text { Costos de } \\
\text { producción } \\
\text { tercer año }\end{array}$ & $\begin{array}{l}\text { Rentabilidad } \\
\text { bruta/ha-año }\end{array}$ \\
\hline \multirow{4}{*}{450} & $d / 3$ & 3 & $1.108,6$ & 2.475 .504 & 1.886 .773 & 218.000 & 370.731 \\
\hline & $d / 3$ & 3 & $1.446,7$ & 3.230 .481 & 1.886 .773 & 338.550 & 1.005 .158 \\
\hline & $d / 4$ & 4 & $1.281,3$ & 2.861 .143 & 1.415 .080 & 338.550 & 1.107.513 \\
\hline & $d / 5$ & 5 & $1.042,9$ & 2.328 .796 & 1.132 .064 & 338.550 & 858.182 \\
\hline \multirow{4}{*}{500} & $d / 3$ & 3,3 & $1.108,6$ & 2.475 .504 & 1.715 .248 & 218.000 & 542.256 \\
\hline & $d / 3$ & 3,3 & $1.446,7$ & 3.230 .481 & 1.715 .248 & 338.550 & 1.176 .683 \\
\hline & $d / 4$ & 4,4 & $1.281,3$ & 2.861 .143 & 1.286 .436 & 338.550 & 1.236.157 \\
\hline & $d / 5$ & 5,5 & $1.042,9$ & 2.328.796 & 1.029 .149 & 338.550 & 961.097 \\
\hline \multirow{4}{*}{600} & $d / 3$ & 4 & $1.108,6$ & 2.475 .504 & 1.415 .080 & 218.000 & 842.424 \\
\hline & $d / 3$ & 4 & $1.446,7$ & 3.230 .481 & 1.415 .080 & 338.550 & 1.476 .851 \\
\hline & $d / 4$ & 5,3 & $1.281,3$ & 2.861 .143 & 1.067 .985 & 338.550 & 1.454 .608 \\
\hline & $d / 5$ & 6,6 & $1.042,9$ & 2.328 .796 & 857.624 & 338.550 & 1.132.622 \\
\hline \multirow{4}{*}{700} & $d / 3$ & 4,6 & $1.108,6$ & 2.475 .504 & 1.230 .504 & 218.000 & 1.027 .000 \\
\hline & $d / 3$ & 4,6 & $1.446,7$ & 3.230 .481 & 1.230 .504 & 338.550 & 1.661 .427 \\
\hline & $d / 4$ & 6,2 & $1.281,3$ & 2.861 .143 & 912.955 & 338.550 & 1.609 .638 \\
\hline & $d / 5$ & 7,7 & $1.042,9$ & 2.328 .796 & 735.106 & 338.550 & 1.255.139 \\
\hline
\end{tabular}

1 Incluye costo estimulación (120.000 \$/ha-año); frecuencias d/3, d/4, d/5.

Costo sangrador $808.617 \$$ (10m/y); precio de venta/kg caucho: 2.900; sistema $10 \mathrm{~m} / \mathrm{y}$ pagado como coagulo de campo oreado con $77 \%$ DRC.

medida que aumenta este rendimiento el mejor sistema es d/4 E, con ganancias superiores a $\$ 1.000 .000$ por ha si se compara al sistema d/3 S.E. Gonçalves et al. (2000) al realizar el análisis económico de diferentes sistemas de sangría en 10 clones evaluados, y con base en 5 años de producción, observaron que la ganancia liquida de algunos clones, tales como el GT 1 y PB 235 en el sistema 1/2S d/4 ET 5\%, son superiores en 12 y $54 \%$, respectivamente, en relación al sistema $1 / 2 \mathrm{~S} \mathrm{~d} / 2$. Sin embargo, la alta concentración de ethephon empleada en este estudio ocasionó alta incidencia de seca fisiológica, teniendo que en la actualidad recomendar la concentración de ET 2,5\% para reducir este efecto.

\section{CONCLUSIONES}

Se recomienda la utilización de sistemas $1 / 2 \mathrm{~S} d / 3$ 6d/7 ET 2,5\% 4/y y 1/2S d/4 6d/7 ET 2,5\% 4/y que poseen similar rentabilidad económica bajo distintas tareas de sangría, siendo esta segunda la que posee menor consumo de corteza y menor incidencia de enfermedades de panel. Esto último hace que se aumente la vida útil de la plantación.

Los mejores contenidos de DRC se obtuvieron con el sistema 1/2S d/3 6d/7 cuando comparado con otros sistemas. Sin embargo, cabe decir que al disminuirse este contenido en el látex se ha encontrado que los sistemas de sangría con estimulación mejoran otras propiedades físicas del látex.

Para realizar los sistemas de sangría con estimulación se requiere una fertilización adecuada que permita que la planta responda al nuevo sistema de explotación.

\section{AGRADECIMENTOS}

Se agradece al Departamento Administrativo de Ciencia y Tecnología (Colciencias) por el financiamiento de esta investigación, a Jorge Posada propietario de la finca El Divino Niño en Barrancabermeja por su colaboración en el desarrollo de la investigación, a la Promotora de Caucho del Magdalena Medio (Procaucho) 
por su apoyo en la investigación a través de su gerente, Hernán Hernández.

\section{REFERENCIAS BIBLIOGRÁFICAS}

Alle, J.Y., E.A. Dick, E.F. Soymahin, R.O. Glaba, J.Z. Keli y S. Obouayeba, S. 2015. Effect of mineral fertilization on agrophysiological parameters and economic viability of clone PB 235 of Hevea brasiliensis in the region of $\mathrm{GO}$ in south western Côte d'Ivoire. J. Anim. Plant Sci. 2, 3768-3780.

Diarrassouba, M., E.F. Soumahin, L.F. Coulibaly, A.E. Badou, K.E. Dick, C. Kouame, S. Obouayeba y S. Ake. 2012. Latex harvesting technologies adapted to clones PB 217 and PR 107 of Hevea brasiliensis Muell. Arg. of the slow metabolism class and to the socio-economic context of Côte d'Ivoire. Int. J. Biosci. 2(12), 125-138.

Di Rienzo, J.A., F. Casonoves, M.G. Balzarini, L. Gonzalez, M. Tablada y C.W. Robledo. 2015. InfoStat version 2015. Grupo InfoStat, FCA, Universidad Nacional de Córdoba, Córdoba, Argentina.

Gonçalves, P., S.R. de Souza, A.P. Brioschi, A. Virgens Filho, A. May y R.S. Alarcon. 2000. Efeito da freqüência de sangria e estimulação no desempenho produtivo e econômico de clones de seringueira. Pesqui. Agropecu. Bras. 35(6), 1081-1091. Doi: 10.1590/ S0100-204X2000000600003

Njukeng, J.N., P.M. Muenyi, B.K. Ngane y E.E. Ehabe. 2011. ethephon stimulation and yield response of some hevea clones in the humid forests of southwest Cameroon. Int. J. Agron. Article ID 257340. Doi: 10.1155/2011/257340

Obouayeba, S., E.F. Soumahin, K.M. Okoma, A.E.B. N’Guessan, R. Lacote, L.F. Coulibaly y S. Aké. 2011. Relationship between the tapping cut length and the parameters of vegetative growth and rubber yield of Hevea brasiliensis, clones GT 1 and PB 235 in south-eastern Côte d'Ivoire. J. Crop Sci. 2(2), 27-44.

Phakagrong, A., P. Chitprasert, S. Liengprayoon, N. Musigamart y L. Vaysse. 2010. Influence of ethephon stimulation on natural rubber properties. En: Proceedings of the $48^{\text {th }}$ Kasetsart University Annual Conference, 3-5 Feb. Bangkok, Tailandia.

Rodrigo, V., K. Kudaligama, K. Fernando y P. Yapa. 2011. Harvesting the rubber tree once in four days: a solution to current issues in the rubber industry in Sri Lanka. J. Rubber Res. Inst. Sri Lanka 91, 15-35.

Silva, J.Q., P. Gonçalves, J.A. Scarpare Filho y R.B. da Costa. 2010. Agronomical performance and profitability of exploitation systems in four rubber tree clones in São Paulo state. Bragantia 69(4), 843-854. Doi: 10.1590/ S0006-87052010000400009

Soumahin, E.F., S. Obouayeba y P.A. Anno. 2009. Low tapping frequency with hormonal stimulation on Hevea brasiliensis clone PB 217 reduces tapping manpower requirement. J. Anim. Plant Sci. 2(3), 109-117.

Soumahin, E.F., S. Obouayeba, K.E. Dick, D.O. Dogbo y P.A. Anno. 2010. Low intensity tapping systems applied to clone PR 107 of Hevea brasiliensis (Muell. Arg.): Results of 21 years of exploitation in South-eastern Cte dIvoire. Afr. J. Plant Sci. 4(5), 142-150.

Virgens Filho, A., A. Moreira y P.R. Castro. 2001. Efeito da calagem e adubação da seringueira no estado nutricional e produção de borracha seca. Pesqui. Agropecu. Bras. 36(8), 1019-1026. Doi: 10.1590/ S0100-204X2001000800003

Virgens Filho, A., A. Moreira y P.R. Castro. 2003. Características físicas e químicas do látex e crescimento da seringueira em função da calagem e da adubação NPK em dois sistemas de explotação. Ciênc. Agrotec. 27(6), 1237-1245. Doi: 10.1590/S1413-70542003000600005 